\title{
Publisher Correction: Follicular lymphoma
}

Nature Reviews Disease Primers (2019) https://doi.org/10.1038/s41572-019-0140-x Published online 12 December 2019

In the originally published version of the PrimeView, the incidence of follicular lymphoma was incorrectly reported as 2,000 new cases diagnosed each year in Europe. This has now been corrected to 2,000 new cases diagnosed each year in France and in the UK. The corrected PrimeView has been added to the HTML and PDF versions of the article.

https://doi.org/10.1038/s41572-020-00217-2 I Published online: 14 September 2020

๑) Springer Nature Limited 2020 